\title{
Reliability and Connectivity Analysis of Vehicluar Ad Hoc Networks for a Highway Tunnel
}

\author{
Saajid Hussain ${ }^{1}$, Di Wu*², Wang Xin ${ }^{3}$, Sheeba Memon ${ }^{4}$, Naadiya Khuda Bux ${ }^{5}$, Arshad Saleem ${ }^{6}$ \\ School of Computer Science and Engineering, Dalian University of Technology Liaoning, Dalian 116024, China ${ }^{1,2}$ \\ School of Mechanical Engineering, Dalian University of Technology Liaoning, Dalian 116024, China ${ }^{3}$ \\ School of Information Science and Engineering, Central South University, Hunan, Changsha 410083, China ${ }^{4,5}$ \\ Shaheed Benazir Bhutto University, Shaheed Benazirabad, Landhi-Nawabshah 67450, Pakistan ${ }^{6}$
}

\begin{abstract}
Vehicular ad-hoc network (VANET) uses 'mobile internet' to facilitate the communication between vehicles and with the goal to ensure road safety and achieve secure communication. Thus the reliability of this type of networks is of paramount significance. The safety-related messages are disseminated in VANETs, on the wireless medium through vehicle to vehicle (V2V) and Vehicle to roadside (V2R) communications. Hence, the Reliability of network is an essential requirement. This paper considers the effect of vehicle transmission range $R_{\text {tran }}$ and vehicle density $\rho$ on the connectivity probability. In addition, a reliability model which takes into account minimal safe headway $S_{h}$ among nearby vehicles at highway tunnel is specified. The reason is that under the tunnel Global Positioning System (GPS), a component of onboard unit $(\mathrm{OBU})$ needs a rich line of sight for perfect services, because due to signal interference, the GPS does not work properly. Though, in the case of a fully connected network, there are chances of danger between vehicles which are close to each other. Therefore, the network is not safe, as accidents and collision can happen at any time. Hence, maintaining the minimal safe headway distance under the tunnel is interesting and useful for VANET. The obtained results show that the little difference of the minimal safe headway under the tunnel can cause a serious change in the entire network reliability. Suggesting that while designing the network reliability models the safe headway $S_{h}$ cannot be ignored.
\end{abstract}

Keywords-Minimal safe headway; reliability; highway tunnel; vehicular ad hoc networks; connectivity probability

\section{INTRODUCTION}

Several studies [1-4], have been conducted to examine the reliability of networks in terms of connectivity, according to the network topology. The reliability of networks is gaining importance day by day particularly safety-sensitive networks like Vehicular ad hoc Networks. In networks, reliability refers to the probability that there prevail no less than one possible connection amongst two vehicles under the specified condition. Reliability issues appear increasingly essential as advance networks turn to be increasingly unpredictable. This encouraged the research on the reliability of networks, which drew much attention during previous decades. In the work of [5], the authors have analyzed vehicle to vehicle wireless connectivity by using mathematical models of mobility and its relation with time. The effect of headway distance, acceleration, association time (i.e. connection setup time), relative speed of vehicles, transmission range and message/data size in short range based $\mathrm{V} 2 \mathrm{~V}$ communications in the models were analyzed. The authors in [6], presented a fluid dynamic model to study dynamism of VANET under various traffic conditions, connectivity between OBUs was analyzed. The authors in [7], proposed an analytical model in which the traffic congestion was avoided by reducing vehicles speed instead of blocking the flow of traffic.

VANETs offer an appropriate environment regarding roadsafety applications. VANET can support two kinds of applications: 1) to support road safety, and 2) to support nonsafety applications. Safety-related applications share necessary information amongst vehicles such as weather conditions, road accident information and propagate emergency messages. Nonsafety related applications provide entertainment to passengers, such as onboard media. These applications are supported by vehicle to vehicle $(\mathrm{V} 2 \mathrm{~V})$ and vehicle to roadside (V2R) communications. Unfortunately, the links between these two communications are not stable enough to achieve optimal performance of these applications. However, because of the dynamic nature of VANETs, the links can live for a short time. The vehicles high mobility causes quick distinctions in the network topology that leads to dynamic changes of the link connectivity. Similarly, the deviation in number of vehicles and the rapid moving vehicles both causes change in the topology, which degrades the network reliability. That is why the network reliability is necessary for both of the communications $\mathrm{V} 2 \mathrm{~V}$ and $\mathrm{V} 2 \mathrm{R}$ and its applications as it is challenging to communicate with each other once the link is unstable.

Several researchers were focusing on the issues of network connectivity in VANETs. For measuring the impacts of network connectivity, the authors in [8], presented empirical research on various speed models and for better estimation of connectivity, Gaussian distribution of the speed perform well. The authors in [9-10], proposed a connectivity probability model based on platoons considering MAC protocols. The proposed model separated the vehicles into two groups, normal and vehicles with platoons and the model was employed to design MAC protocol. The research conducted in [11-12] presented an infrastructure supported network connectivity considering two-channel models: log-normal shadowing and unit disk communication model. The authors in [11] analyzed the uplink and downlink connectivity probabilities. 
Besides, it has been proved by the authors in [13], that the probability of having no isolated node and the probability of having a connected network asymptotically meets a similar value. The results of the study in [14], suggested that a slight change in communication range results in a major difference in connectivity probability in the case of high vehicle density. Further, to maintain the connectivity of vehicles transmission range $R$ plays an essential role [15-16]. Mostly, a larger range of communication results in a large area covered within transmission range which helps to enhance link connectivity performance as compared to small transmission range.

The studies discussed above are interesting. Especially the research conducted by the authors in [17], vehicles are supposed to work with vehicular cloud networks in which videos and pictures for certain vehicles are taken by its neighboring vehicles and transferred via cloud to centralized cloud server. The recommended vehicular cloud network could not include roadside units (RSUs) since their availability is not possible in road tunnels. Hence, messages takes more than two hops in this network resulting in an increasing localization delay.

Though the researchers above did not consider the minimum safe distance under the highway tunnel among nearby vehicles, and only focused on the network connectivity. The reliability of the network plays an essential role in message dissemination on the road regarding entertainment and safety applications. Though, in the case of a fully connected network, there are chances of danger between vehicles which are close to each other. Therefore, the network is not safe, as accidents and collision can happen at any time. Thus, for the analysis of network reliability problems, the minimal safe distance amongst vehicles under the highway tunnel was considered in this study.

In this paper, a network reliability model based on safe distance under the tunnel has been proposed, which provide a possible method to overcome the issues in existing schemes.

The rest of the paper is organized as follows. Section 2 give details of the derived reliability model under the tunnel, which consider the impact of minimum safe headway amongst adjacent vehicles. Section 3 provides the performance evaluation and Section 4 concludes the paper.

\section{SAFE HEAdWAy BASEd RELIABILITy MODEL}

\section{A. System Design}

In VANETs, due to the dynamic movement of vehicles, the links can live for a short time because of the short association time between vehicles. Moreover, because of the road design the links are also restricted. Hence, the message propagation is mostly transmitted over V2V and V2R multi-hop communications. Therefore, the topology of the links plays an essential role in propagating messages successfully. Instead, we can say, a vehicle connection can have a direct impact on message dissemination. The arrival time of vehicles in VANETs follows exponential distribution with parameter $\lambda$ with a flow of traffic $\lambda$ veh/sec [18]. Consider an $L \mathrm{~km}$ of tunnel with two-lane segment of road for the analysis perspective, where every vehicle arriving the tunnel with a random speed $v_{i}$ at 0 , and exits at $L$, as given in the Fig. 1.

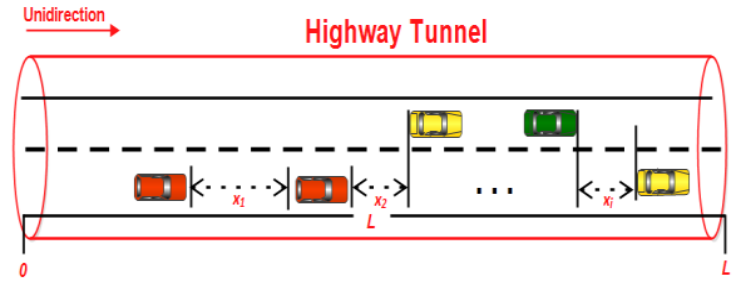

Fig. 1. Tunnel Model.

Evaluation of distance between vehicles in a transit system is called headway [19], represented as $x_{i}, i=1,2, \ldots, N$ refer to Fig. 1. According to the literature, the headway distance is exponentially distributed $\mathrm{i}, \mathrm{i}, \mathrm{d}$ with parameter $\beta$ Therefore, the CDF of headway is $\mathrm{X}=\mathrm{x}$

$F_{X}(x)=1-e^{-\beta x}$

It should be noted that in free flow state the various speed of vehicles is a Gaussian distribution [20]. For safe driving in the tunnel, the two limits are defined for speed $v_{i} \in\left[v_{\min }, v_{\max }\right]$. Hence, a truncated Gaussian (PDF) could be defined as

$g(v)=\frac{f(v)}{\int_{v_{\min }}^{v_{\max }} f(v) d v}$

Where $f(v)=\frac{1}{\delta \sqrt{2 \pi}} e\left(-\frac{(v-\mu)^{2}}{2 \delta^{2}}\right)$ is a Gaussian PDF with the standard deviation $\delta$ and mean speed $\mu$. As the area under the normal curve of the speeds in range $(\mu-3 \delta, \mu-3 \delta)$ is $99.74 \%$ of the whole area [19]. Hence, it is adequate to assign $v_{\min }=\mu-3 \delta$ and $v_{\max }=\mu+3 \delta$ as $\min$ and $\max$ speed of the vehicle's.

Therefore $g(v)$ could be written as

$g(v)=\frac{2 f(v)}{\operatorname{erf}\left(\frac{v_{\max }-\mu}{\delta \sqrt{2}}\right)-\operatorname{erf}\left(\frac{v_{\min }-\mu}{\delta \sqrt{2}}\right)}$

In the above equation $\operatorname{erf}($.) represents the error function. Therefore, the values of expected speed could be calculated as

$E(V)=\int_{v_{\min }}^{v_{\max }} v g(v) d v$

Where the average density of vehicles in the tunnel is defined as

$\rho=\frac{1}{E[Y]}=\lambda E\left[\frac{1}{V}\right]$

Finally, the number of average vehicles traveling in a tunnel segment $L$ is calculated as

$N_{e}=L \rho$

The number of maximum vehicles that could be available on a given segment of tunnel with length $L$ is defined as [12]

$N_{t}=\frac{L}{S_{h}} N_{L n}$

Where $S_{h}$ represents the minimal safe headway distance among vehicles, and $N_{L n}$ is the total number of tunnel lanes. Hence, the normalized vehicle density can be estimated as

$K_{e}=\frac{N_{e}}{N_{t}}$ 


\section{B. Reliability based on Safe Headway Distance}

The two vehicles could be considered connected if the transmission range is greater than the distance between them. Based on traffic theory, the $\beta=\rho$. As stated in eq. (1), it can be written as reliability of two connected vehicles.

$R_{v 2 v}=1-e^{-\rho R_{\text {tran }}}$

In the above equation, $R_{\text {tran }}$ represents the transmission range for all vehicles. In order to guarantee the safety between vehicles, the minimal headway distance would be considered. By setting $S_{h}$ as a safe minimal headway distance amongst vehicle, the reliability amongst every two vehicles could be defined as

$R_{v 2 v}=1-e^{-\rho\left(R_{\text {tran }}-S_{h}\right)}$

To guarantee the safety for all the vehicles $N_{e}$ in the tunnel segment $L$, it is required that $x_{i} \leq R_{\text {tran }}-S_{h}$ for $i=$ $1,2, \ldots, N_{e}-1$. As stated earlier, $x_{i}$ are $i . i . d$, so the reliability of the network is connected within the tunnel segment $L$ could be defined as

$R_{n e t}=\left(1-e^{-\rho\left(R_{\text {tran }}-S_{h}\right)}\right)^{\left(N_{e}-1\right)}$

The equation given above is used to compute the network reliability within one-hop communication. Hence, $R_{\text {tran }}$ which is the one-hop transmission range could be achieved from Eq. (11), as given below

$R_{\text {tran }}=S_{h}-\frac{1}{\rho} \log _{e}\left(1-N^{-1} \sqrt{R_{\text {net }}}\right)$

Let the minimum value of $R_{\text {net }}$ be $95 \%$, with no loss of generality, $R_{\min }$ which is the minimum one-hop transmission range is defined as

$R_{\text {min }}=S_{h}-\frac{1}{\rho} \log _{e}\left(1-N_{e}-1 \sqrt{0.95)}\right.$

\section{EXPERIMENTAL RESULTS AND ANALYSIS}

Considering the above analysis, the reliability performance is discussed, by taking into account the minimal safe headway distance in the tunnel. The numerical results are discussed in this section. The MATLAB is used to perform the simulations. The simulated scenario is given in Fig.1, which is based on a one-way two-lane road segment tunnel with $10 \mathrm{~km}$ length.

\section{A. The Average Density of Vehicles against Arrival Rate}

The relation amongst the average density of vehicles and the arrival rate under various $\mu$ with constant $\delta=5 \mathrm{~m} / \mathrm{s}$ is given in Fig. 2 and 3. The speed of all vehicles follows a truncated Gaussian distribution, in the simulation as defined in eq. (2) and (3). The interrelation amongst the average density of vehicles and arrival rate of vehicles under various $\mu$ while constant $\delta=5 \mathrm{~m} / \mathrm{s}$ is given in Fig. 2 .

Fig. 3 shows the interrelation of Average density of vehicles and the arrival rate under various $\delta$ with constant $\mu=$ $35 \mathrm{~m} / \mathrm{s}$.

Obviously, with the increasing arrival rate $\lambda$ the average density of vehicles is increased when the rate of the $\mu$ and $\delta$ are constant. Moreover, with the same arrival rate of vehicles, the higher average speed $\mu$ will cause higher density of vehicles, where, higher standard deviation value $\delta$ results in low density. It is clear that, higher density of vehicles enhance network reliability in terms of connectivity. So as a conclusion by considering Fig. 2 and 3, it can be written as: by increasing $\mu$ the average density of vehicles will be improved and decreased by the increase in $\delta$.

\section{B. Reliability against Transmission Range}

Fig. 4 shows the interrelation amongst reliability and transmission range $R_{\text {tran }}$.

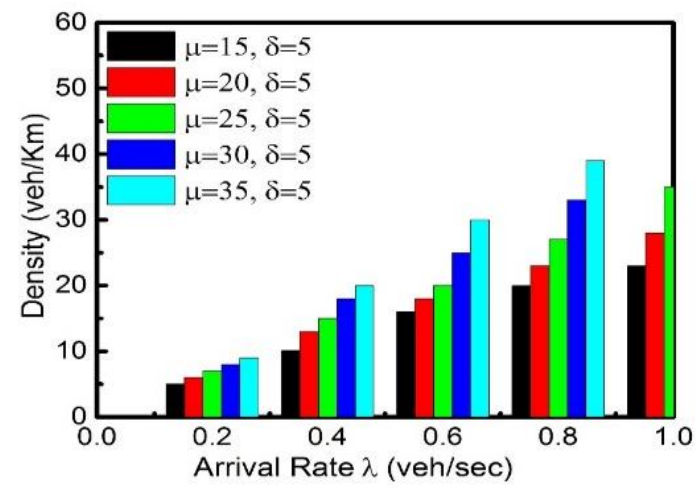

Fig. 2. Average density of vehicles against vehicle arrival rate with $\delta=$ $5 \mathrm{~m} / \mathrm{s}$

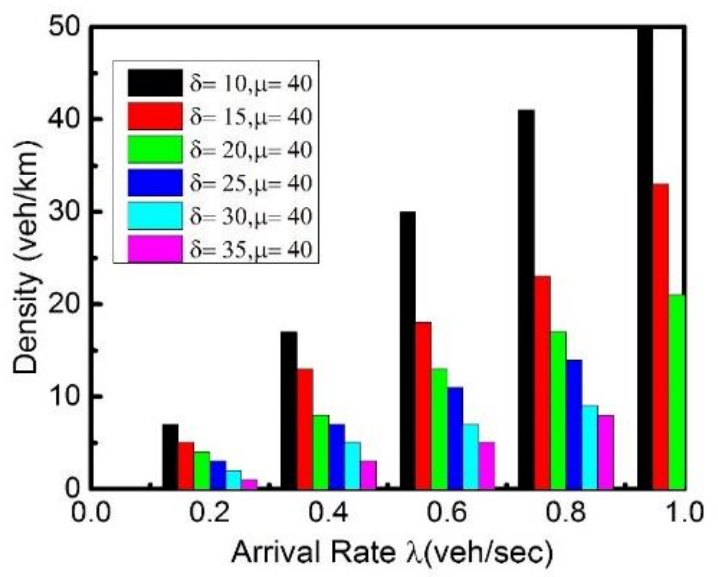

Fig. 3. Average density of vehicles against vehicle arrival rate when $\mu=35 \mathrm{~m} / \mathrm{s}$.

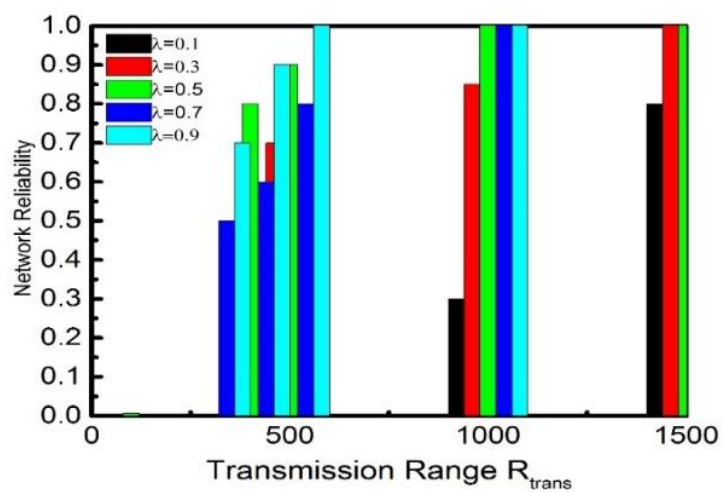

Fig. 4. Reliability against transmission range $R_{\text {tran }}(\mu=20 \mathrm{~m} / \mathrm{s}, \delta=$ $5 \mathrm{~m} / \mathrm{s}$ ). 
When $\mu$ and $\delta$ values are constant, the decrease in reliability is observed with the increasing transmission range $R_{\text {tran }}$. In the scenario where transmission range is larger, there are more chances for vehicles to successfully establish links to propagate messages as compared to a smaller range. Moreover, in a given constant $R_{\text {tran }}$, the reliability increases with the increasing arrival rate of vehicles $\lambda$. The higher $\lambda$ shows the more vehicles will enter the desired tunnel segment, and leads to a high density of vehicles. Same as the density of vehicles which were presented in Fig. 2 and 3, surely the reliability in terms of connectivity would be enhanced.

\section{Reliability against Average Density}

Fig. 5 shows the interrelation amongst the reliability and the vehicles average density $\rho$. In a specified transmission range $R_{\text {tran }}$, it is observed that with the increasing density of vehicles reliability increased. In a stated density of vehicles, the reliability increases with the increasing transmission range $R_{\text {tran }}$.

It is clear in Fig. 5, the reliability touches to 1 when $R_{\text {tran }}$ approach to $500 \mathrm{~m}$ or larger, If the density of vehicles is greater than $0.01 \mathrm{veh} / \mathrm{km}$ (which shows 100 vehicles in the segment of $10000 \mathrm{~m}$ tunnel). That could be said, that the aim is achieved and network becomes fully reliable, also with high successful probability, the message could be propagated. However, a substantial variation in the reliability has been observed when $R_{\text {tran }}=100 \mathrm{~m}$ or $<100 \mathrm{~m}$. The reliability remained zero, even the density of vehicles reached to $0.04 \mathrm{veh} / \mathrm{Km}$.

The analysis conducted above shows that when transmission range $R_{\text {tran }}$ is small such as $100 \mathrm{~m}$, even a trivial change in $R_{\text {tran }}$ results in a worst performance of entire network reliability. Where, in VANETs, network reliability is an important factor which affects the network performances. Next, we highlight the point that the minimal headway distance amongst vehicle as well results in a great effect on the entire network reliability while transmission range is small.

\section{The Minimal Safe Headway $S_{h}$ and its Effect on the Network Reliability}

Here, setting the minimal distance amongst vehicle as the safe headway $S_{h}$. In Fig. 6 and 7 effects of $S_{h}$ on the network reliability is shown.

It is observed from Fig. 6 and 7 that, a little deviation such as $5 m$ in the $R_{\text {tran }}$, can cause serious variations in the network reliability with various $S_{h}$ values. When $R_{\text {tran }}=90 \mathrm{~m}$ and $R_{\text {tran }}=95 \mathrm{~m}$, the reliability variations are arisen when the density of vehicles ranging from 0.07 to $0.15 \mathrm{veh} / \mathrm{km}$. The changes in the reliability are appeared when $R_{\text {tran }}=100 \mathrm{~m}$ and the density of vehicles ranging from 0.05 to $0.14 \mathrm{veh} / \mathrm{Km}$. When $R_{\text {tran }}=150 \mathrm{~m}$, the changes in the reliability are observed when density of vehicles ranging from $0.03 \mathrm{veh} / \mathrm{Km}$ to $0.07 \mathrm{veh} / \mathrm{Km}$.

As observed, when the $R_{\text {tran }}=150 \mathrm{~m}$, the change in the reliability is not as important as when $R_{\text {tran }}<150 \mathrm{~m}$. As an example we took $R_{\text {tran }}=200 \mathrm{~m}$ in Fig. 7 , under various $S_{h}$ the bars remained almost same. Hence, when $R_{\text {tran }}$ reaching to more than $150 \mathrm{~m}$, the variation in $S_{h}$ would not seriously affect the network reliability, which is shown in Fig. 7.

\section{E. Discussions}

As Global Positioning System (GPS), which is the component of onboard unit (OBU) needs a rich line of sight for perfect services because of the signal interference the GPS do not work properly. Though, in the case of a fully connected network, there are chances of danger between vehicles which are close to each other. Therefore, the network is not safe, as accidents and collision can happen at any time. Hence, maintaining the minimal safe headway distance under the tunnel is interesting and useful for VANET. It can be observed from the Fig. 6 and 7 the impact of $S_{h}$ on the network reliability when the vehicles communication range is small, specifically when $R_{\text {tran }}<150 m$.

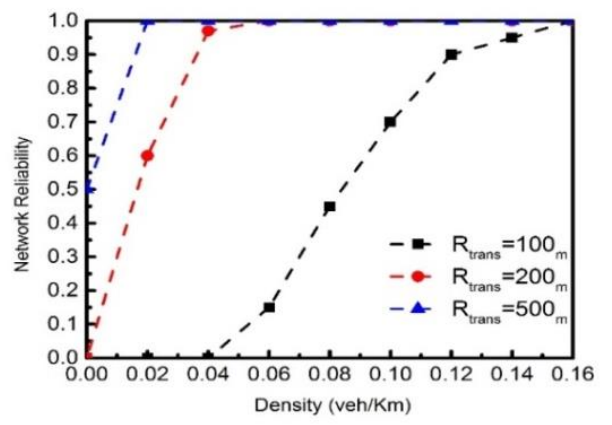

Fig. 5. Reliability Against Average Density of Vehicles.
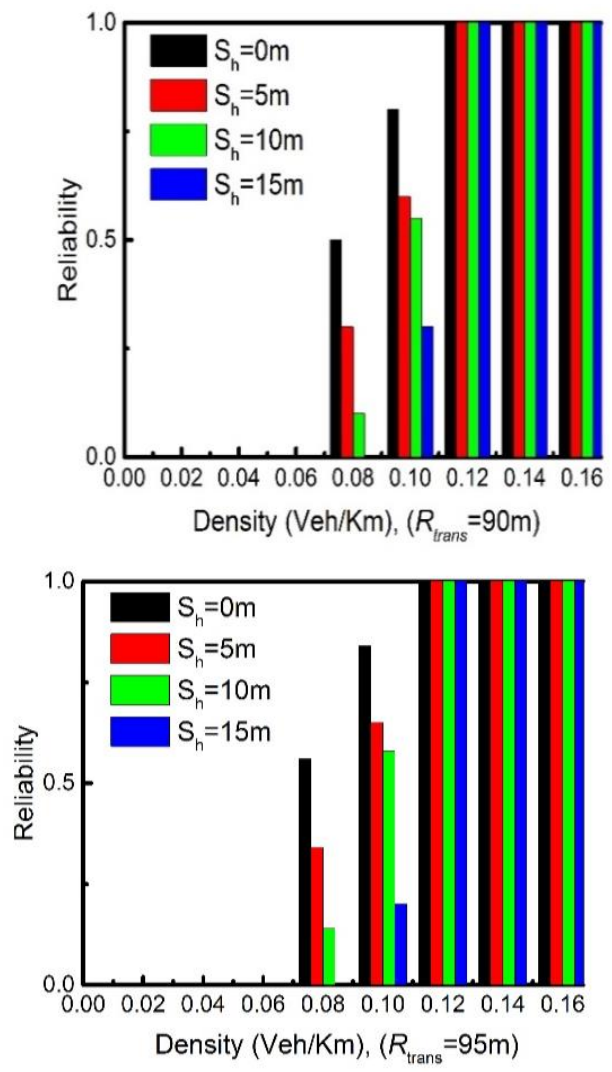

Fig. 6. The Impacts of $S_{h}$ on the Reliability While $R_{\text {tran }}=90 \mathrm{~m}$ and $95 \mathrm{~m}$. 

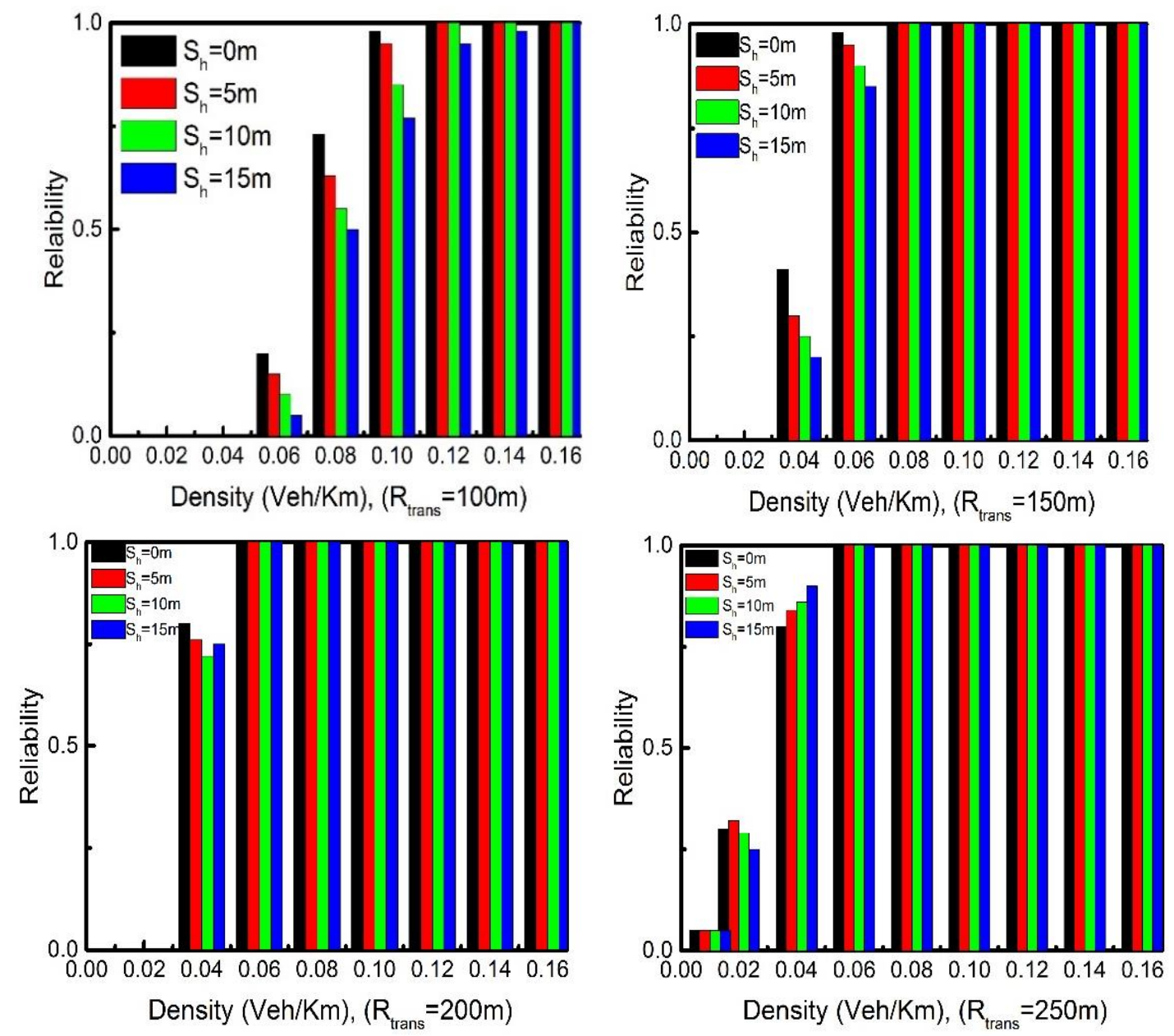

Fig. 7. The Impacts of $S_{h}$ on the Reliability While $R_{\text {tran }}=100 \mathrm{~m}, 150 \mathrm{~m}, 200 \mathrm{~m}$ and $250 \mathrm{~m}$.

\section{CONCLUSION}

Vehicular ad hoc networks support two kinds of communications $\mathrm{V} 2 \mathrm{~V}$ and $\mathrm{V} 2 \mathrm{R}$, which are mainly used to propagate messages of entertainment and safety applications. Hence, the main feature for the successful propagation of message is link connectivity. Generally, the transmission range, density of vehicles, and the arrival rate of vehicles will affect the network reliability performance. In this paper, we design a reliability calculation scheme which considers the influence of the minimal safe distance amongst nearby vehicles under the tunnel. The results obtained by simulations prove that the deviation of the safe distance will bring substantial changes in the network reliability when transmission range of vehicles is less than $150 \mathrm{~m}$. Hence, while designing the network reliability models, the safe headway cannot be ignored.

\section{ACKNOWLEDGMENT}

This work was supported by the National Natural Science Foundation of China (no. 61370201) and the Open Research Found from the Key Laboratory for Computer Network and Information Integration (Southeast University, Ministry of Education, China).

\section{REFERENCES}

[1] Dharmaraja, Selvamuthu, Resham Vinayak, and Kishor S. Trivedi. "Reliability and survivability of vehicular ad hoc networks: An analytical approach." Reliability Engineering \& System Safety 153 (2016): 28-38.

[2] Zhao, Tong, et al. "A Link Reliability Model of Metropolitan VANETs for Data Dissemination." Vehicular Technology Conference (VTC-Fall), 2016 IEEE 84th. IEEE, 2016.

[3] Ahmed, Waqar, et al. "Reliability modeling and analysis of communication networks." Journal of Network and Computer Applications 78 (2017): 191-215.

[4] Ma, Xiaomin, and Kishor S. Trivedi. "Reliability and performance of general two-dimensional broadcast wireless network." Performance Evaluation 95 (2016): 41-59.

[5] Yan, Gongjun, and Danda B. Rawat. "Vehicle-to-vehicle connectivity analysis for vehicular ad-hoc networks." Ad Hoc Networks 58 (2017): 25-35.

[6] Umer, Tariq, et al. "Modeling vehicles mobility for connectivity analysis in vanet." Intelligent Transportation Systems. Springer International Publishing, 2016.

[7] Hussain, S.; Wu, D.; Memon, S.; Bux, N.K. Vehicular Ad Hoc Network (VANET) Connectivity Analysis of a Highway Toll Plaza. Data 2019, 4, 28.

[8] S. M. Abuelenin, A. Y. Abul-Magd, "Empirical study of traffic velocity distribution and its effect on VANETs connectivity", Proc. of 2014 ICCVE, pp. 391-395, 2014. 
[9] C. Shao, S. Leng, B. Fan, Y. Zhang, A. Vinel, M. Jonsson, "Connectivity-aware Medium Access Control in platoon-based Vehicular Ad Hoc Networks", Proce. of 2015 IEEE ICC, pp. 3305-3310, 2015.

[10] C. Shao, S. Leng, Y. Zhang, A. Vinel, M. Jonsson, "Performance Analysis of Connectivity Probability and Connectivity-Aware MAC Protocol Design for Platoon-Based VANETs", IEEE Trans. on Vehicu. Tech, vol. 64, no. 12, pp. 5596-5609, Dec. 2015.

[11] S. C. Ng, W. Zhang, Y. Zhang, Y. Yang, G. Mao, "Analysis of Access and Connectivity Probabilities in Vehicular Relay Networks", IEEE Journal on Selected Areas in Communications, vol. 29, no. 1, pp. 140150, January 2011.

[12] S. C. Ng, and G. Mao, "Analysis of k-hop Connectivity Probabilities in 2-D Wireless networks with Infrastructure support,v in Proc. of 2010 IEEE Globecom.

[13] X. Ta, G. Mao, Y. Zhang, B. D. O. Anderson, "On the connectivity of wireless Multi-hop Networks with arbitrary wireless channel models", IEEE Communications Letters, vol. 13, no. 3, pp. 181-183, March 2009.

[14] J. Wu, "Connectivity Analysis of a Mobile Vehicular Ad Hoc Network with Dynamic Node Population", Proc. of 2008 IEEE Globecom Workshops, pp. 1-8, 2008.
[15] X Luo, G Liu, H Huang, "Buffer Capacity-Constrained Epidemic Routing Model in Mobile Ad-Hoc Networks", proc. of IEEE International Conference on Computational Science and Engineering, pp. 1443-1448, 2014.

[16] H Yao, H Huang, Q Liang et al., "The Effect of Critical Transmission Range in Epidemic Data Propagation for Mobile Ad-hoc Social Network" in Pervasive Computing and the Networked World, Berlin Heidelberg:Springer, pp. 743-756, 2013.

[17] Agarwal, Y. , Jain, K. , Kumar, S. , \& Bhardwaj, G. N. . (2016). TLST: Time of Arrival Based Localization and Smart Tunnel concept in VANETs. 2016 3rd International Conference on Signal Processing and Integrated Networks (SPIN). IEEE.

[18] S. Yousefi, E. Altman, R. El-Azouzi, and M. Fathy, "Improving connectivity in vehicular ad hoc networks: An analytical study," Computer communications, vol. 31, no. 9, pp. 1653-1659, 2008.

[19] Yousefi S, Altman E, El-Azouzi R, et al. "Analytical model for connectivity in vehicular ad hoc networks," IEEE Trans. Veh. Technol., vol. 57 , no. 6 , pp. 3341-3356, Nov 2008.

[20] R. Roess, E. Prassas, and W. McShane, Traffic Engineering, 2010. 